\title{
Evolution of a very complex active region during the decay phase of Cycle 23
}

\author{
Mariano Poisson ${ }^{1,2}$, Marcelo López Fuentes ${ }^{1,2}$, \\ Cristina H. Mandrini ${ }^{1,2}$, Pascal Démoulin ${ }^{3}$, \\ Etienne Pariat $^{3}$ \\ ${ }^{1}$ Instituto de Astronomía y Física del Espacio (CONICET-UBA), \\ CC 67 Suc 28, 1428 Buenos Aires, Argentina \\ ${ }^{2}$ Facultad de Ciencias Exactas y Naturales, \\ UBA, Buenos Aires, Argentina \\ ${ }^{3}$ Observatoire de Paris, LESIA, 92195 Meudon, France
}

\begin{abstract}
We study the emergence and evolution of AR NOAA 10314, observed on the solar disk during March 13-19, 2003. This extremely complex AR is of particular interest due to its unusual magnetic flux distribution and the clear rotation of the polarities of a $\delta$-spot within the AR. Using SOHO/MDI magnetograms we follow the evolution of the photospheric magnetic flux to infer the morphology of the structure that originates the AR. We determine the tilt angle variation for the $\delta$-spot and find a counter-clockwise rotation corresponding to a positive writhed flux tube. We compute the magnetic helicity injection and the total accumulated helicity in the $\mathrm{AR}$ and find a correlation with the observed rotation.
\end{abstract}

Keywords. Sun: photosphere, Sun: magnetic fields

\section{Introduction}

It has been shown in previous studies that magnetically complex solar active regions (ARs) present the higher rates of flare production and coronal mass ejections (CMEs) (Liu et al. 2005). Magnetic flux distributions that form $\delta$-spots at photospheric level are among these cases (Linton et al. 1998). These structures are related to the emergence of magnetic flux tubes that have been highly distorted by the effect of convective turbulence beneath the photosphere (Fan 2009). For this reason, it is expected to find high magnetic helicity injection rates in these particular ARs. The accumulation of magnetic helicity in the solar atmosphere is related to the energy release mechanisms, since a large amount of free magnetic energy is present in highly stressed magnetic structures. Recent studies have pointed at these peculiar ARs as the sources of the stronger geomagnetic storms (Szajko et al., this volume).

Here, we study how the complex photospheric evolution of AR 10314 is consistently related to a high magnetic helicity injection. In Section 2 we describe the observed photospheric motion of the 4 main polarities. In Section 3 we compute and analyze the evolution of the tilt angle of the $\delta$-spot and the magnetic helicity injected in the AR. In Section 4 we discuss the flux tube morphologies that can qualitatively reproduce the observed evolution of the polarities and quantitatively explain the amount of magnetic helicity calculated in Section 3 . 

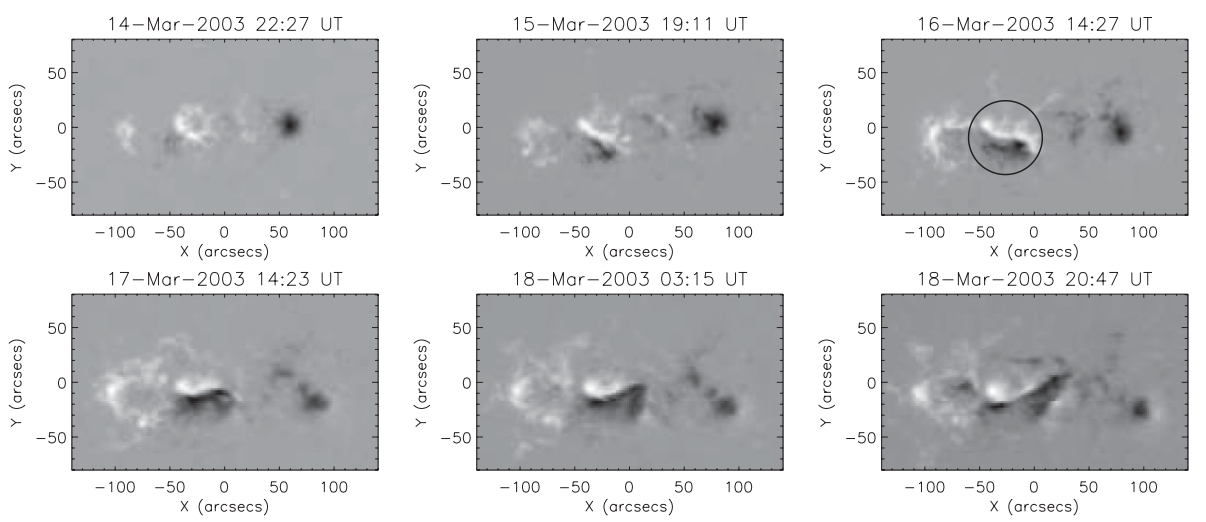

Figure 1. Selected SOHO/MDI magnetograms for the evolution AR 10314. The AR was observed from its first emergence on March 132003 to its disappearance on the west limb around March 20 2003. In the upper right panel we indicate with a circle the location of the $\delta$-spot formed at the center of the AR. The main polarities of the $\delta$-spot are observed to rotate one around the other during the studied evolution.

\section{Photospheric evolution}

We follow the photospheric evolution and the coronal activity of the studied AR using full disk longitudinal magnetograms taken with the Michelson Doppler Imager (MDI, see Scherrer et al. 1995) on board the Solar Heliospheric Observatory (SOHO).

The AR NOAA 10314 emerged near the solar disk center (S15W18) on March 13, 2003. It evolved from a dual-bipolar system to a more complex configuration with the appeareance of a $\delta$-spot at its center (marked with a circle in the upper right panel of Figure 1). A feature that is of particular interest for this work is the constant rotation of the inversion line of the $\delta$-spot (see the lower row panels of Figure 1 ).

\section{Analysis}

\subsection{Evolution of the $\delta$-spot tilt}

Using MDI magnetograms we compute the flux weighted mean positions of the positive and negative polarities of the $\delta$-spot (see López Fuentes et al. 2003). From these positions we compute the tilt angle as the angle that the vector $\mathbf{S}$ joining both polarities (from positive to negative) form with the East-West direction.
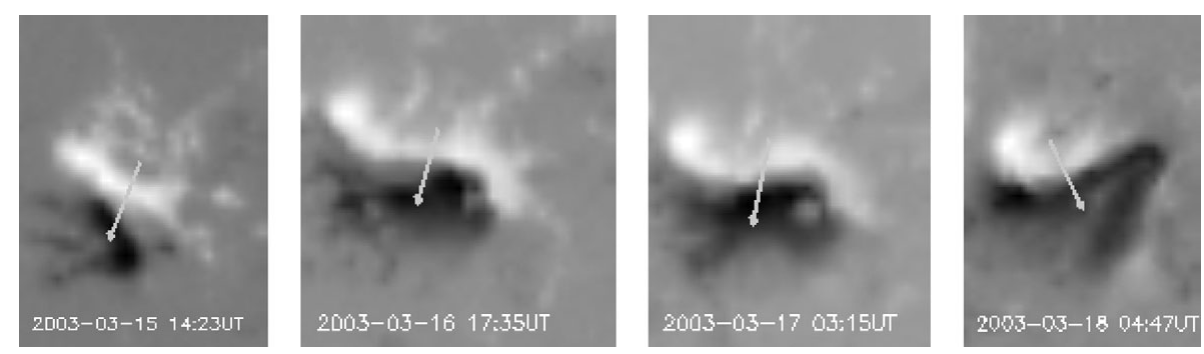

Figure 2. Selected MDI magnetograms of the $\delta$-spot in AR 10314. The ploted vectors represent the relative position of the positive and negative polarities.

Figure 2 shows the tilt angle variation of the $\delta$-spot by displaying the vector $\mathbf{S}$ for selected frames. Our results show a sustained counter-clockwise rotation of the polarities. From the observed rotation we are able to infer that the writhe of the associated magnetic flux tube, as well as the main helicity flux contribution are positive. 


\subsection{Magnetic helicity injection}

We analyze the injection of magnetic helicity during the AR evolution following the procedure described in Pariat et al. (2005). We use the Differential Affine Velocity Estimator (DAVE) routine developed by Schuck $(2005,2006)$ in order to find the velocity field, from which we can in turn compute the magnetic helicity density. In figure 3 we show selected maps of magnetic helicity density. The density maps show a significant positive helicity injection in the area of the $\delta$-spot region. The integration of the helicity density for the full AR confirms the dominance of positive helicity injection and is consistent with the observed counter-clockwise rotation of the tilt angle (Poisson et al. 2011).
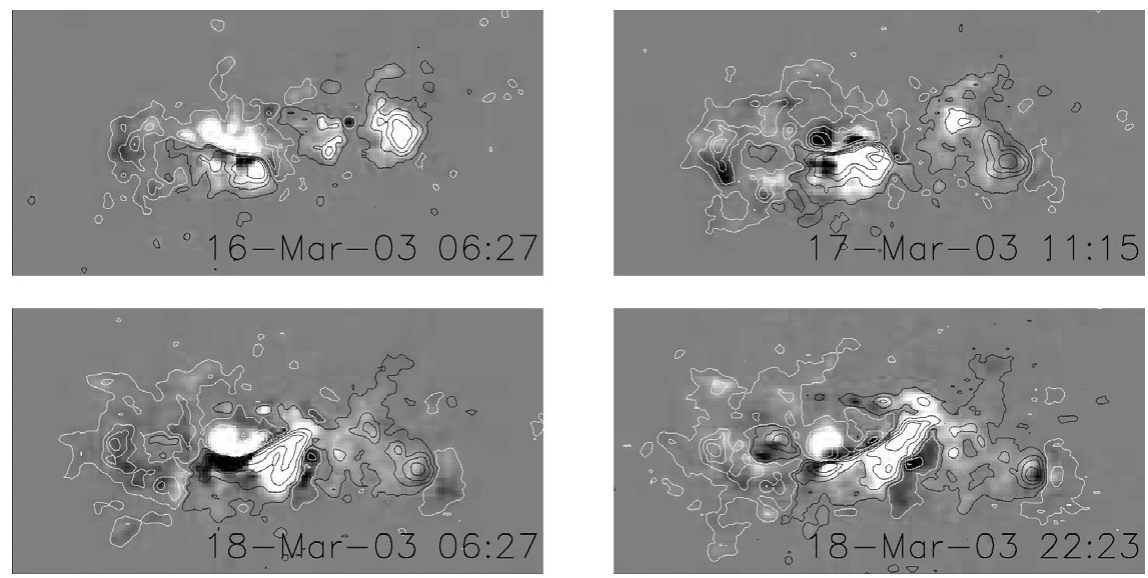

Figure 3. Magnetic helicity density maps for AR 10314. White and black intensities over the map correspond to positive and negative helicity flux contributions. Overlayed white and black contours correspond to positive and negative photospheric magnetic field strengths of 100, 600, 1200 and $1800 \mathrm{G}$.

\section{Discusion}

We devise two possible models for the magnetic structure that produced AR 10314 that are consistent with the photospheric field distribution and the evolution of the tilt angle. The first one (see Figure 4, left panel) corresponds to a single deformed flux tube that presents a kink instability, and the second one (Figure 4, right panel) is formed by two independent flux tubes.
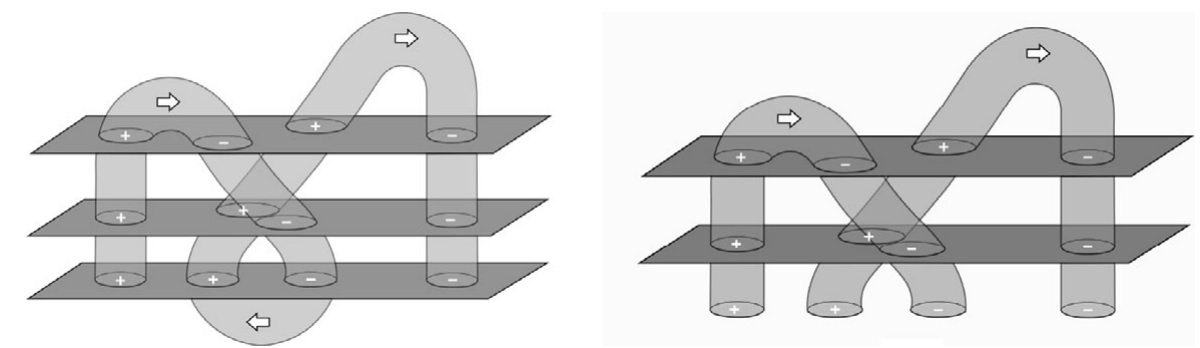

Figure 4. Possible structures of the magnetic flux tube that produced AR 10314. The planes indicate the relative position of the photosphere during the flux tube emergence, the arrows show the global direction of the magnetic field along the tube, and the circles with signs correspond to the location of the polarities observed at the photospheric plane for different stages of the evolution. 
Although the method used to compute the magnetic helicity does not allow us to discard any of these two posibilities, the continuous rotation of the tilt angle and the constant distance between the main polarities of the $\delta$-spot, strongly support the first scenario (single flux tube with a kink).

\section{Conclusions}

We study the evolution of AR 10314 from a dual-bipole to a complex configuration with the development of a $\delta$-spot. We propose two different models of the flux tube morphology in order to explain the observed AR photospheric evolution. One of the models corresponds to a flux tube with a kink instability and the other to two independent flux tubes. We study the evolution of the tilt angle and observe a counter-clockwise rotation of the $\delta$-spot with a constant distance between its main polarities. These results are consistent with the emergence of a positive writhed flux tube and support the single flux-tube model. Finally, we analyze the magnetic helicity injection during the AR evolution and find an overwhelming dominance of positive helicity, which is also consistent with the above results (for further analysis see Poisson et al. 2011).

\section{Acknowledgements}

MLF and CHM are members of the Carrera del Investigador Científico of the Consejo Nacional de Investigaciones Científicas y Técnicas (CONICET), Argentina. The authors would like to thank Dr. Peter Schuck for the permission to use the Differential Affine Velocity Estimator (DAVE) routine. SOHO is a project of international cooperation between ESA and NASA.

\section{References}

Fan, Y. 2009, Living Reviews in Solar Physics, 6, 4

Linton, M. G., Dahlburg, R. B., Fisher, G. H., \& Longcope, D. W. 1998, ApJ, 507, 404

Liu, C., Deng, N., \& Liu, Y. et al. 2005, ApJ, 622, 722

López Fuentes, M. C., Démoulin, P., Mandrini, C. H., Pevtsov, A. A., \& van Driel-Gesztelyi, L. 2003, A\& $A, 397,305$

Pariat, E., Démoulin, P., \& Berger, M. A. 2005, A\& A, 439, 1191

Poisson, M., López Fuentes, M., Mandrini, C. H., Démoulin, P., \& Pariat, E. 2011, submitted to Adv. Space Res.

Scherrer, P. H., Bogart, R. S., Bush, R. I. et al. 1995, Sol. Phys., 162, 129

Schuck, P. W. 2005, ApJ, 632, L53

Schuck, P. W. 2006, ApJ, 646, 1358

Szajko, N., Cristiani, G., Mandrini, C. H., \& Dal Lago, A. 2011, this volume 